Simultaneous Determination of the Source-Time Function and Reflectivity via Inversion

Susan E. Minkoff

William W. Symes

October, 1993

TR93-44 



\title{
Simultaneous Determination of the Source-Time Function and Reflectivity via Inversion
}

\author{
Susan E. Minkoff* William W. Symes*
}

June 23, 1993

\begin{abstract}
No attempt to uncover the desired mechanical properties of the earth will be successful in reflection seismology without an accurate estimate of the source term in the wave equation model. In this paper we argue for using inversion to solve the source calibration problem while at the same time discerning the earth parameters. We show both mathematically and through a numerical example how highly effective inversion can be in this determination when reproducible sources provide redundancy in the seismic data.
\end{abstract}

\section{Introduction}

The term "model" implies that one has made simplifications that capture some essential characteristics of the real situation while making the problem more tractable. In reflection seismology, the simplifying assumption that the source is known (measured in a lab) is not valid. The mechanical properties of the earth, the understanding of which is the goal of reflection seismology, are coupled with the action of the source in the data. Many techniques have been used to estimate the action of the source such as statistical methods or measurement of the direct wave. (See [Ziolkowski, 1984], for a discussion of these methods.) Others have determined both the source and reflectivity simultaneously via inversion (see [Bube et al., 1988]; [Lewis, 1989]; [Sacks, 1990]). (Further examples were presented in the talk by [Kolb and Canadas, 1986].) However, all of these inversion results rely on assumptions which prevent actual application. Our current work focuses on the inversion of the source and reflectivity using models which plausibly describe reflection seismic data.

The underlying assumption in this work is that one ought to be able to separately determine both the source and reflectivity functions due to inherent redundancy in the

*The Rice Inversion Project, Department of Computational and Applied Mathematics, Rice University, Houston TX 77251-1892 
data. This redundancy arises in situations where the sources are reproducible (such as with air gun sources in marine seismology) (see [Dragoset et al., 1987]). In such cases, the data traces differ only in the incidence angles at which these identical sources illuminate a given section of the earth.

This paper describes the model, assumptions, and problem and discusses past work on simultaneous determination of the source and reflectivity using inversion. A numerical result is given which indicates that inversion might indeed succeed in determining both the source and reflectivity.

\section{Model and problem specifications}

We model the earth as an acoustic fluid with constant density and variable sound velocity c. The velocity is assumed to vary slowly on the scale of a seismic wavelength and to determine the kinematics of wave propagation. The short-scale heterogeneities are modeled by the relative perturbation in the velocity, or the reflectivity, $r$. Mathematically, we write $r(\vec{x})=\delta c(\vec{x}) / c(\vec{x})$. The source is assumed to be isotropic and to have, approximately, point support.

By assuming a primaries only, or single-scattering, approximation and by using highfrequency asymptotics, one may derive the multi-dimensional convolutional model which is a high-frequency approximation to the seismogram (see [Beylkin, 1985]).

$$
\begin{aligned}
& S\left(\overrightarrow{x_{s}}, \overrightarrow{x_{r}}, t_{r}\right)=f\left(t_{r}\right) * \tilde{r}\left(\overrightarrow{x_{s}}, \overrightarrow{x_{r}}, t_{r}\right), \text { where } \\
& S=\text { the seismogram, } \\
& f=\text { the source wavelet. } \\
& \vec{x}_{s}=\text { the spatial source location, } \\
& \vec{x}_{r}=\text { the spatial receiver location, } \\
& t_{r}=\text { time. }
\end{aligned}
$$

Here "*" denotes convolution in time. The reflectivity as a function of time, $\tilde{r}$ (or perturbation of the Green's function for the acoustic wave equation) is given by

$$
\begin{aligned}
& \tilde{r}\left(\overrightarrow{x_{s}}, \overrightarrow{x_{r}}, t_{r}\right) \approx \int d \vec{x}\left[A\left(\overrightarrow{x_{s}}, \overrightarrow{x_{r}}, \vec{x}\right)\left(N\left(\overrightarrow{x_{s}}, \overrightarrow{x_{r}}, \vec{x}\right) \cdot \nabla\right)^{2} r\left(\vec{x}, t_{r}\right)\right] \\
& \times \delta\left(t_{r}-\tau\left(\overrightarrow{x_{s}}, \vec{x}\right)-\tau\left(\overrightarrow{x_{r}}, \vec{x}\right)\right) \quad \text { where }
\end{aligned}
$$

$$
A=\text { the reflectivity amplitude from geometric optics, }
$$




$$
\begin{aligned}
& \tau\left(\overrightarrow{x_{s}}, \vec{x}\right)=\text { the travel-time from } \overrightarrow{x_{s}} \text { to } \vec{x}, \\
& \tau\left(\overrightarrow{x_{r}}, \vec{x}\right)=\text { the travel-time from } \overrightarrow{x_{r}} \text { to } \vec{x}, \\
& N=\text { the unit outward normal to the surface, }
\end{aligned}
$$

$$
N=-\left(\nabla_{x} \tau\left(\overrightarrow{x_{s}}, \vec{x}\right)+\nabla_{x} \tau\left(\overrightarrow{x_{r}}, \vec{x}\right)\right)
$$

This expression is approximate up to a smoother error term. Thus, for high frequency sources, this most singular term gives the greatest contribution to the seismogram.

If, moreover, we make the assumption that the model is of a layered medium, then the sound velocity, $c$, and reflectivity, $r$, vary only with depth, $z \equiv x_{3}$. By applying the Radon integral transform (or plane-wave decomposition) to the normal displacement gotten from the solution to the acoustic wave equation, one can reduce the three-dimensional problem to a family of one-dimensional equations (see [Treitel et al., 1982]). These equations are parameterized by slowness, $p$. In this setting, we may rewrite the equation for the seismogram as

$$
S(t, p)=f(t) * \tilde{r}(t, p)
$$

where now the expression for the reflectivity is given by

$$
\tilde{r}(t, p) \approx \int d z[A(z) r(z, p)] \delta(t-2 \tau)
$$

and the travel-time function, $\tau$, can be written

$$
\tau(z, p)=\int_{0}^{z} d \zeta \frac{\sqrt{1-c^{2}(\zeta) p^{2}}}{c(\zeta)}
$$

This primaries-only, plane-wave, layered medium, constant density acoustic model is likely the simplest model of seismic wave propagation which one can use to describe real seismograms. For an example of its use in modeling seismic field data see [Symes and Carazzone, 1991].

\section{Mathematical results}

\subsection{The constant background velocity case - general results}

Perhaps the most realistic theoretical results to date for the separate determination of the source and reflectivity via inversion were presented by Lewis in his thesis ([Lewis, 1989]). 
He analyzed the effect of small perturbations in the source and reflectivity on the seismogram, in the case that the background velocity is assumed constant. While the seismogram is linear in the source and reflectivity separately, it is nonlinear in these parameters collectively. From the plane-wave convolutional model for the seismogram, we may write the perturbed seismogram as

$$
\delta S(t, p)=\delta f(t) * \tilde{r}(t, p)+f(t) * \delta \tilde{r}(t, p)
$$

Lewis showed that the perturbed seismogram determines both the perturbation in the source and the perturbation in the reflectivity uniquely. For instance, if the perturbation in the data is equal to zero, then the perturbations in both the source and reflectivity must also be identically zero. Obvious nonuniqueness due to scale ambiguities between the two parameters (source and reflectivity) is ruled out by an added constraint which fixes the scale of one of the two quantities.

Further, he showed that perturbations in the seismic data stably determine corresponding perturbations in the source and reflectivity. This determination for source perturbations is valid within the passband of the source itself. Similarly, determination of reflectivity perturbations is constrained to the spatial frequency passband for the reflectivity. The stability improves as the range of apertures increases. Conversely, as the range is reduced to a single trace, the ability to separately determine the perturbations in the source and reflectivity simultaneously is lost.

As is realistic, in this work Lewis assumed that both the source and reflectivity have compact support in time. Thus, the source cannot mathematically be truly band-limited. It is reasonable, however, to assume that outside of some frequency band the source has small enough contribution to be considered negligible and ignored (see [Slepian, 1983]).

Lewis' result implies that a local optimization or descent method, applied to a misfit error function, will converge to the correct source and reflectivity provided that the initial guess of these two parameters is sufficiently accurate. It does not provide information about the global behavior of misfit error functions, nor does it give explicit numerical stability bounds. These matters must be investigated computationally.

\subsection{The stability estimate}

In an effort to generalize the above result to the case in which the background velocity need not be constant, we have arrived at a new proof of the fact that the seismic data stably determines corresponding perturbations in the source and reflectivity. The theorem below still applies, however, to the constant background velocity case. 
Theorem 3.1 If the scale of the source time function is fixed, i.e. if

$$
\|\hat{f}\|_{L^{2}\left(\Omega_{\omega}\right)}^{2}=1
$$

and we consider source data in the passband of $f$ (which for seismic sources excludes frequencies in an interval around zero),

$$
|\omega \hat{f}(\omega)| \geq \eta>0
$$

and if the norm of the reflectivity function over large enough frequency intervals is always nonzero, namely,

$$
\int_{\Omega_{\xi}} d \xi|\hat{r}(\xi)|^{2} \geq \epsilon\|\hat{r}\|_{L^{2}}^{2}>0, \quad\left|\Omega_{\xi}\right|>\left|\Omega_{\min }\right|
$$

Then, the perturbations in the seismogram stably determine corresponding perturbations in the source and reflectivity functions:

$$
\|\widehat{\delta f}\|_{L^{2}\left(\Omega_{\omega}\right)} \leq K\|\widehat{\delta S}\|_{L^{2}\left(\Omega_{\omega} \times\left[0, p_{\max }\right]\right)}
$$

and

$$
\int_{\frac{\sqrt{1-c^{2} p_{\max }^{2}}}{c} \Omega_{l}}^{\frac{1}{\Omega_{u}}} d \eta|\widehat{\delta r}(\eta)|^{2} \leq K\|\widehat{\delta S}\|_{L^{2}\left(\Omega_{\omega} \times\left[0, p_{\max }\right]\right)}
$$

Proof: From the previous section we have an expression for the perturbed seismogram which we Fourier Transform

$$
\widehat{\delta S}(\omega, p)=\widehat{\delta f}(\omega) \hat{\tilde{r}}(\omega, p)+\hat{f}(\omega) \widehat{\delta \tilde{r}}(\omega, p)
$$

In the constant background velocity case, the reflectivity function is

$$
\widehat{\tilde{r}}(\omega, p)=\frac{c}{\sqrt{1-c^{2} p^{2}}} \hat{r}\left(\omega \frac{\sqrt{1-c^{2} p^{2}}}{c}\right)
$$


and the effective velocity is

$$
v(p)=\frac{c}{\sqrt{1-c^{2} p^{2}}}
$$

Substituting expressions numbered 3.7 and 3.8 into equation 3.6 gives

$$
\widehat{\delta S}(\omega, p)=\widehat{\delta f}(\omega) v(p) \hat{r}\left(\frac{\omega}{v(p)}\right)+\hat{f}(\omega) v(p) \widehat{\delta r}\left(\frac{\omega}{v(p)}\right)
$$

Multiplying this expression by $\overline{\hat{r}}(\omega /(v(p)))$ we get

$$
\frac{\overline{\hat{r}}\left(\frac{\omega}{v(p)}\right)}{v(p)} \frac{\widehat{\delta S}(\omega, p)}{\hat{f}(\omega)}=\left[\frac{\widehat{\delta f}(\omega)}{\hat{f}(\omega)}\right] \hat{r}\left(\frac{\omega}{v(p)}\right) \overline{\hat{r}}\left(\frac{\omega}{v(p)}\right)+\widehat{\delta r}\left(\frac{\omega}{v(p)}\right) \overline{\hat{r}}\left(\frac{\omega}{v(p)}\right)
$$

Now change variables, letting $\xi=(\omega /(v(p)))$ and $d \xi=\left(\omega v^{\prime}(p) d p\right) / v^{2}(p)$.

By equation 3.8 we see that

$$
d \xi=\frac{\omega p c}{\sqrt{1-c^{2} p^{2}}} d p
$$

Choosing a finite set of points $\left\{\omega_{i}\right\}$ which are ordered so that $\omega_{i}>\omega_{i+1}, i=1, \ldots, N$, we see that for fixed $i$ the new variable $\xi$ must be contained in the range

$$
\frac{\omega_{i}}{v\left(p_{\max }\right)} \leq \xi \leq \frac{\omega_{i}}{v\left(p_{\min }\right)}
$$

In the expression above, $\xi$ varies as $p$ takes on values in the range $p \epsilon\left(p_{\text {min }}, p_{\text {max }}\right)$.

Looking at the first two points in the set $\left\{\omega_{i}\right\}$, we integrate the difference between equation 3.10 at the point $\omega_{1}$ and equation 3.10 at $\omega_{2}$ against the factor in expression 3.11 .

$$
\begin{gathered}
\frac{1}{\omega_{1} \hat{f}\left(\omega_{1}\right)} \int_{\Omega_{1}} \xi d \xi \overline{\hat{r}}(\xi) \widehat{\delta S}\left(\omega_{1}, \frac{\sqrt{\omega_{1}^{2}-c^{2} \xi^{2}}}{c \omega_{1}}\right)- \\
\frac{1}{\omega_{2} \hat{f}\left(\omega_{2}\right)} \int_{\Omega_{1}} \xi d \xi \overline{\hat{r}}(\xi) \widehat{\delta S}\left(\omega_{2}, \frac{\sqrt{\omega_{2}^{2}-c^{2} \xi^{2}}}{c \omega_{2}}\right)=\left[\frac{\widehat{\delta f}\left(\omega_{1}\right)}{\hat{f}\left(\omega_{1}\right)}-\frac{\widehat{\delta f}\left(\omega_{2}\right)}{\hat{f}\left(\omega_{2}\right)}\right] \int_{\Omega_{1}} d \xi|\hat{r}(\xi)|^{2} .
\end{gathered}
$$


Here the second argument of the function $\widehat{\delta S}$ is derived from the relation $p=v^{-1}(\omega / \xi)$ where

$$
v^{-1}(x)=\frac{\sqrt{x^{2}-c^{2}}}{c x}
$$

The region of integration $\Omega_{1}$ is an assumed "overlap" between the $\xi$ ranges for $\omega_{1}$ and $\omega_{2}$ :

$$
\Omega_{1} \equiv\left\{\xi: \xi \epsilon\left(\frac{\omega_{1}}{v\left(p_{\max }\right)}, \frac{\omega_{2}}{v\left(p_{\min }\right)}\right)\right\}
$$

By assumption 3.3 we have that the norm of the reflectivity is bounded away from zero so long as $\left|\Omega_{1}\right| \geq\left|\Omega_{\min }\right|$. Note that the closer $\omega_{1}$ is to $\omega_{2}$, the bigger the region of overlap. However, we choose the points in the set $\left\{\omega_{i}\right\}$ to make the difference $\omega_{1}-\omega_{2}$ as large as possible. We wish to cover the passband of the source, $\Omega_{\omega}$. If instead $\left|\Omega_{1}\right|<\left|\Omega_{\text {min }}\right|$, then we may continue to subdivide that region of frequency space by adding points to our set $\left\{\omega_{i}\right\}$ until the requirements of the hypothesis are satisfied. Therefore, renumbering the points in $\left\{\omega_{i}\right\}$ if necessary, we assume $\left|\Omega_{1}\right| \geq\left|\Omega_{m i n}\right|$ and divide through by the norm of the reflectivity, which leads to the expression

$$
\left[\frac{\widehat{\delta f}\left(\omega_{1}\right)}{\hat{f}\left(\omega_{1}\right)}-\frac{\widehat{\delta f}\left(\omega_{2}\right)}{\hat{f}\left(\omega_{2}\right)}\right]=
$$

$\frac{1}{\int_{\Omega_{1}} d \xi|\hat{r}(\xi)|^{2}}\left[\frac{1}{\omega_{1} \hat{f}\left(\omega_{1}\right)} \int_{\Omega_{1}} \xi d \xi \overline{\hat{r}}(\xi) \widehat{\delta S}\left(\omega_{1}, \frac{\sqrt{\omega_{1}^{2}-c^{2} \xi^{2}}}{c \omega_{1}}\right)-\frac{1}{\omega_{2} \hat{f}\left(\omega_{2}\right)} \int_{\Omega_{1}} \xi d \xi \overline{\hat{r}}(\xi) \widehat{\delta S}\left(\omega_{2}, \frac{\sqrt{\omega_{2}^{2}-c^{2} \xi^{2}}}{c \omega_{2}}\right)\right]$

We could perform the same calculations to arrive at equation 3.15 for the two points $\omega_{2}$ and $\omega_{3}$, and so on. In this way we get $N-1$ such difference equations. Thus we see that

$$
\sum_{i=1}^{N-1}\left[\frac{\widehat{\delta f}\left(\omega_{i}\right)}{\hat{f}\left(\omega_{i}\right)}-\frac{\widehat{\delta f}\left(\omega_{i+1}\right)}{\hat{f}\left(\omega_{i+1}\right)}\right]=\sum_{i=1}^{N-1} \frac{1}{\int_{\Omega_{i}} d \xi|\hat{r}(\xi)|^{2}}
$$

$\times\left[\frac{1}{\omega_{i} \hat{f}\left(\omega_{i}\right)} \int_{\Omega_{i}} \xi d \xi \overline{\hat{r}}(\xi) \widehat{\delta S}\left(\omega_{i}, \frac{\sqrt{\omega_{i}^{2}-c^{2} \xi^{2}}}{c \omega_{i}}\right)-\frac{1}{\omega_{i+1} \hat{f}\left(\omega_{i+1}\right)} \int_{\Omega_{i}} \xi d \xi \overline{\hat{r}}(\xi) \widehat{\delta S}\left(\omega_{i+1}, \frac{\sqrt{\omega_{i+1}^{2}-c^{2} \xi^{2}}}{c \omega_{i+1}}\right)\right]$

The regions of integration in expression 3.16 , namely the $\Omega_{i}$, are all defined analogously to the way in which $\Omega_{1}$ was defined. The sum telescopes, leaving only the first and last terms 
on the left-hand side of equation 3.16. Holding $\omega_{N}$ fixed, $\left(k=\widehat{\delta f}\left(\omega_{N}\right) / \hat{f}\left(\omega_{N}\right)\right)$, varying $\omega_{1}$ (which we have renamed $\omega$ ), and taking the $L^{2}$ inner product of the above expression with $\widehat{\delta f}(\omega)$ we get

$$
\langle\widehat{\delta f}(\omega)-k \hat{f}(\omega), \widehat{\delta f}(\omega)\rangle=\langle D, \widehat{\delta f}(\omega)\rangle
$$

Here " $\mathrm{D}$ " stands for the right-hand side of equation 3.16 (the known data elements). And, the variable $\omega$ now can range over all of $\Omega_{\omega}$.

By linearizing the constraint on the size of $f$, namely hypothesis 3.1 , we get

$$
\int_{\Omega_{\omega}} d \omega \widehat{\delta f}(\omega) \overline{\hat{f}}(\omega)=0
$$

which along with the Cauchy-Schwarz inequality and equation 3.17 implies that

$$
\|\widehat{\delta f}\|_{L^{2}\left(\Omega_{\omega}\right)}^{2} \leq\|D\|_{L^{2}\left(\Omega_{\omega} \times \Omega_{\xi}\right)}\|\widehat{\delta f}\|_{L^{2}\left(\Omega_{\omega}\right)}
$$

Dividing through by $\|\widehat{\delta f}\|_{L^{2}\left(\Omega_{\omega}\right)}$ and using the lemma proved below gives the desired result 3.4 .

The second conclusion of the theorem, that the perturbation in the reflectivity is also bounded by a perturbation in the seismogram comes from equation 3.9 and the estimate just proved for the perturbation in the source. For details see Lewis' thesis.

Lemma 3.2 Under the same hypotheses as in the theorem, namely 3.1, 3.2, and 3.3, we have that

$$
\|D\|_{L^{2}\left(\Omega_{\omega} \times \Omega_{\xi}\right)} \leq\|\widehat{\delta S}\|_{L^{2}\left(\Omega_{\omega} \times\left[0, p_{\max }\right]\right)}
$$

where

$$
\begin{gathered}
D \equiv \sum_{i=1}^{N-1} \frac{1}{\int_{\Omega_{i}} d \xi|\hat{r}(\xi)|^{2}} \\
\times\left[\frac{1}{\omega_{i} \hat{f}\left(\omega_{i}\right)} \int_{\Omega_{i}} \xi d \xi \overline{\hat{r}}(\xi) \widehat{\delta S}\left(\omega_{i}, \frac{\sqrt{\omega_{i}^{2}-c^{2} \xi^{2}}}{c \omega_{i}}\right)-\frac{1}{\omega_{i+1} \hat{f}\left(\omega_{i+1}\right)} \int_{\Omega_{i}} \xi d \xi \overline{\hat{r}}(\xi) \widehat{\delta S}\left(\omega_{i+1}, \frac{\sqrt{\omega_{i+1}^{2}-c^{2} \xi^{2}}}{c \omega_{i+1}}\right)\right] .
\end{gathered}
$$


Proof: It suffices to examine the behavior of one of the two factors in the difference expression $\mathrm{D}$ for one term in the sum. By repeated application of the Cauchy-Schwarz inequality we see that

$$
\begin{gathered}
\frac{1}{\int_{\Omega_{\xi}} d \xi|\hat{r}(\xi)|^{2}} \int_{\Omega_{\omega}} \int_{\Omega_{\xi}} d \xi d \omega\left[\xi \overline{\hat{r}}(\xi) \frac{1}{\omega \hat{f}(\omega)} \widehat{\delta S}\left(\omega, \frac{\sqrt{\omega^{2}-c^{2} \xi^{2}}}{c \omega}\right)\right] \\
\leq \frac{1}{\|\hat{r}\|_{L^{2}\left(\Omega_{\xi}\right)}^{2}}\left|\left\langle\frac{\xi \bar{r}(\xi)}{\omega \hat{f}(\omega)}, \widehat{\delta S}\left(\omega, \frac{\sqrt{\omega^{2}-c^{2} \xi^{2}}}{c \omega}\right)\right\rangle\right| \\
\leq \frac{|\xi|}{\|\hat{r}\|_{L^{2}\left(\Omega_{\xi}\right)}} \frac{\|\widehat{\delta S}\|_{L^{2}\left(\Omega_{\omega} \times\left[0_{1} p_{\max }\right]\right)}}{\|\omega \hat{f}(\omega)\|_{L^{2}\left(\Omega_{\omega}\right)}}
\end{gathered}
$$

The result follows from the three hypotheses.

\section{A numerical example}

To test the theoretical results discussed above numerically, we performed experiments on synthetic data generated from real data. The example shown here was created from Gulf of Mexico data we received from Exxon Production Research Company. All tests were done on a Sun Sparcstation 2 using the Differential Semblance Optimization package, a seismic simulation and inversion code under development at Rice University.

We began with one common-midpoint data gather taken from a marine seismic experiment done of this area. The Radon transform was applied to yield 48 plane-wave traces with slowness values ranging from $p_{\min }=.1158 \mathrm{~ms} / \mathrm{m}$ to $p_{\max }=.36468 \mathrm{~ms} / \mathrm{m}$. We were also given an estimate of the anisotropic air gun source in the form of a 31-term Legendre expansion in slowness. The coefficients in the series were functions of time only.

The procedure used to generate the synthetic model for our experiment was the following. First, a $15 \mathrm{~Hz}$ Ricker filter was applied to both the source and the plane-wave data to allow us to use a coarser sampling rate. The target isotropic source is the first trace of the 31-trace source estimate. This isotropic source has peak frequency of roughly 15 $\mathrm{Hz}$ and a peak in time at $110 \mathrm{~ms}$. To generate a realistic target reflectivity, we inverted the plane-wave field data for the reflectivity using a smoothed version of a well-log for the background velocity. The output of the (least-squares) inversion was an estimate of the reflectivity (the relative perturbation in the velocity in this case).

For the experiment reported here (determination of the source and reflectivity) we chose a constant background velocity of $2.1 \mathrm{~m} / \mathrm{ms}$ which was an approximate mean velocity from the well-log. We then generated new data for our synthetic experiment using the 
reflectivity from the field data inversion as input. The seismic data consisted of 13 traces each of about 3 seconds time duration.

The inversion for source and reflectivity was performed rising a method known as coordinate search or alternation. The method follows.

Repeat until convergence:

1. Given the current source, $f_{c}$, and current reflectivity, $r_{c}$, invert for a new estimate of the reflectivity $r_{+}$.

2. Replace $r_{c}$ by $r_{+}$.

3. Given the current source and reflectivity guesses, $f_{c}, r_{c}$, invert for a new estimate of the source $f_{+}$.

4. Replace $f_{c}$ by $f_{+}$.

Alternation, although notoriously inefficient, is attractive for initial experiments because it requires only successive solution of simple linear least-squares problems. Obviously, quasi-Newton methods could be applied to the problem and would likely reduce the number of iterations dramatically.

The initial estimate of the reflectivity used for the inversions was $r=0$. For the initial source estimate, we chose a Ricker wavelet also with peak frequency of $15 \mathrm{~Hz}$ but which had its peak centered in time at $0 \mathrm{~ms}$. The source location was of interest to us in this experiment. In practice, one might reasonably expect to be able to estimate the power spectrum of the source. However, one would not expect to be able to guess at the location of the peak in time (i.e., the phase). Each inversion round included an estimation of the source and an estimation of the reflectivity. In all, 60 rounds of inversions were performed to reduce the root mean square error to $5 \%$ of the data norm.

Figure 4.1 shows the synthetically-generated data which our experiment attempts to match during the inversion jobs. Figure 4.2 shows the target reflectivity and final inversion result. The fit is so close, one has difficultly telling that two graphs have been plotted. Figure 4.3 shows the initial source guess. Figures 4.4 and 4.5 show the movement of the source to the target source location. Figure 4.6 shows the target source and final inversion result. As was discussed in the mathematical results section, an obvious nonuniqueness of solutions exists when using the convolutional model to determine both the source and reflectivity parameters. Scaling the source up by a constant $\alpha$ and the reflectivity down by $1 / \alpha$ results in the same fit to the seismic data. Thus, for display purposes, a normalization was done to adjust the the amplitudes of the graphs in figures 4.2 and 4.6. The maximum amplitudes of the target source and reflectivity functions were used to scale the final estimated source and reflectivity functions, respectively. 


\section{Conclusion}

In reflection seismology, an accurate estimate of the source function which generated a particular set of seismic data is crucial to accurate recovery of the mechanical properties of the earth. When data is generated from a reproducible source, redundancy in this data provides hope that separate determination of the source and reflectivity may be possible. A simple but useful model of seismograms is given by the plane-wave convolutional model derived from the constant density, variable sound velocity acoustic wave equation. Our numerical result suggests inversion may be useful for realistic determination of the source and reflectivity. The source location in time need not be known a priori for the true location and source signature to be recovered. The assumption of constant background velocity used in Lewis' work and in this numerical example, however, is unrealistic. Constant background velocity models do not represent accurately enough the kinematics of seismic wave propagation. We have run experiments which indicate that recovery of the source and reflectivity is indeed possible in the case of a band-limited source and variable background velocity. We believe we can extend the stability theory to this case, thus ensuring a sound basis for simultaneous determination of the source and earth properties via inversion. Further, we conjecture that extensions of the theory and algorithm will accommodate anisotropic sources, nonlayered subsurface geometry, and more complex physics (e.g., viscoelasticity).

Acknowledgments: This work was partly supported by the National Science Foundation, the Office of Naval Research, the State of Texas, and the sponsors of The Rice Inversion Project: Advance Geophysical, Amoco Research, Conoco Inc., Mobil Research and Development Corp., Exxon Production Research Co., Earth Modeling Systems, and Cray Research Inc. We are grateful to Exxon Production Research Co. and James J. Carazzone for provision of a plane-wave field data set, and to Catherine Lewis for preparation of a well $\log$.

This work is an expanded version of a paper which will appear in the Society of Exploration Geophysicists' 1993 Annual International Meeting Abstract Book.

\section{References}

[Beylkin, 1985] Beylkin, G. (1985). Imaging of discontinuities in the inverse scattering problem by inversion of a causal generalized radon tranform. J. Math. Phy., 26:99-108.

[Bube et al., 1988] Bube, K., Lailly, P., Sacks, P., Santosa, F., and Symes, W. W. (1988). Simultaneous determination of source wavelet and velocity profile using impulsive pointsource reflections from a layered fluid. Geophysical Journal, 95:449-462.

[Dragoset et al., 1987] Dragoset, B., Hargreaves, N., and Larner, K. (1987). Air-gun source instabilities. Geophysics, 52(9):1229-1251. 
[Kolb and Canadas, 1986] Kolb, P. and Canadas, G. (1986). Least-squares inversion of prestack data: Simultaneous identification of density and velocity of stratified media. In 56th Annual International SEG Meeting, pages 604-607, Houston, TX, USA. Society of Exploration Geophysicists.

[Lewis, 1989] Lewis, R. M. (1989). Source-Velocity Identification for a Layered Model of Reflection Seismology. PhD thesis, Rice University.

[Sacks, 1990] Sacks, P. E. (1990). A velocity inversion problem involving an unknown source. SIAM J. Appl. Math., 50(3):931-941.

[Slepian, 1983] Slepian, D. (1983). Some comments on fourier analysis, uncertainty and modeling. SIAM Review, 25(3):379-393.

[Symes and Carazzone, 1991] Symes, W. W. and Carazzone, J. J. (1991). Velocity inversion by differential semblance optimization. Geophysics, 56:654-663.

[Treitel et al., 1982] Treitel, S., Gutowski, P. R., and Wagner, D. E. (1982). Plane-wave decomposition of seismograms. Geophysics, 47:1375-1401.

[Ziolkowski, 1984] Ziolkowski, A. (1984). Deconvolution. International Human Resources Development Corporation, Boston. 


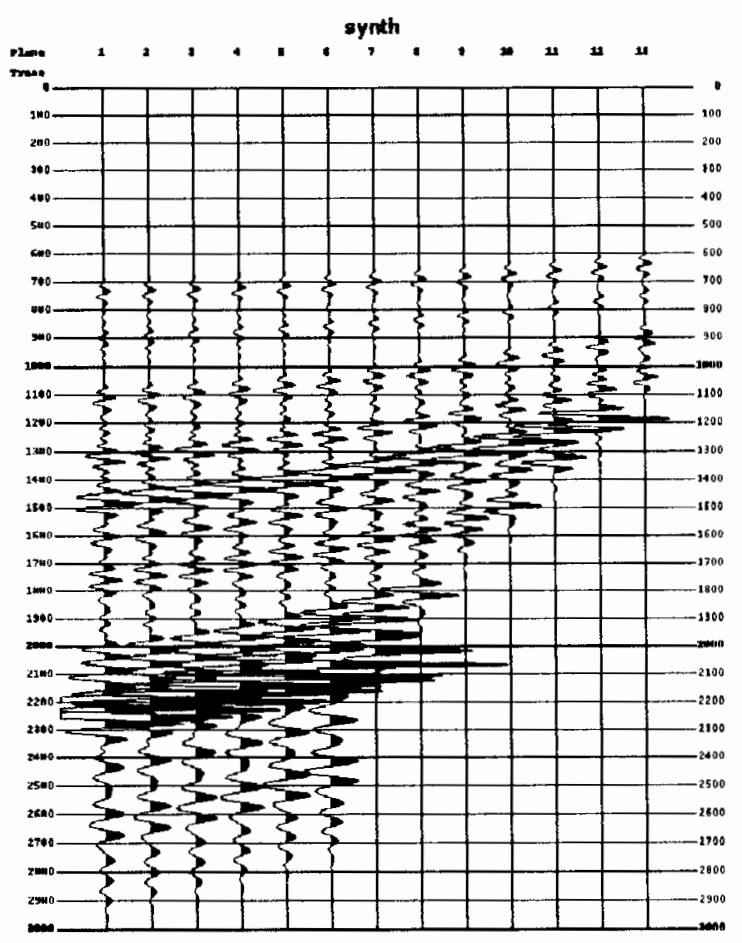

Figure 4.1: Seismic data generated from a constant background velocity of $2.1 \mathrm{~m} / \mathrm{ms}$, and the isotropic source shown in figure 4.6. Three seconds worth of data was used for this experiment. 


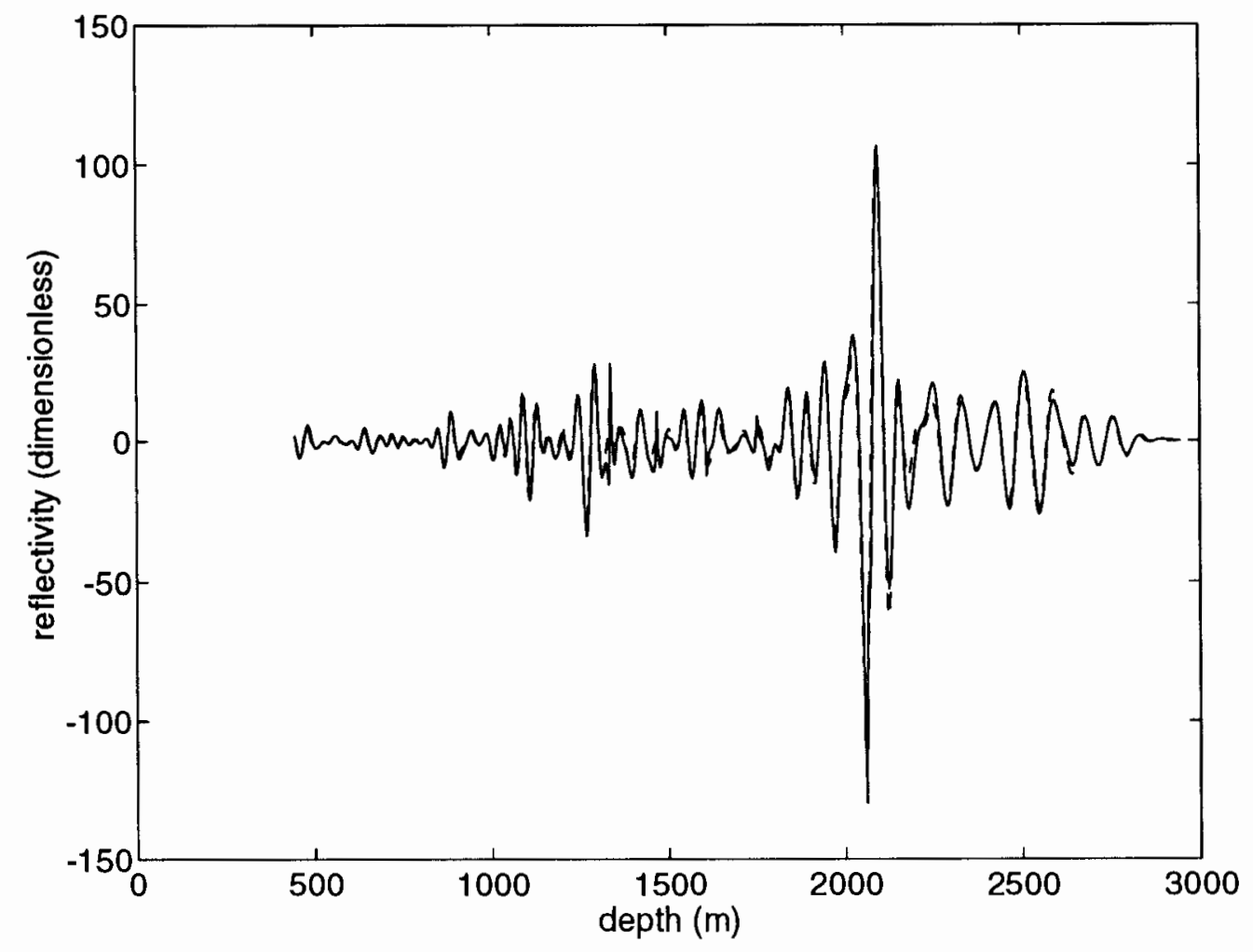

Figure 4.2: Solid line: Final inversion result for reflectivity (iteration round 60). Dashed line: target reflectivity. The final reflectivity estimate was scaled so that the peak amplitudes for both graphs corresponded. 


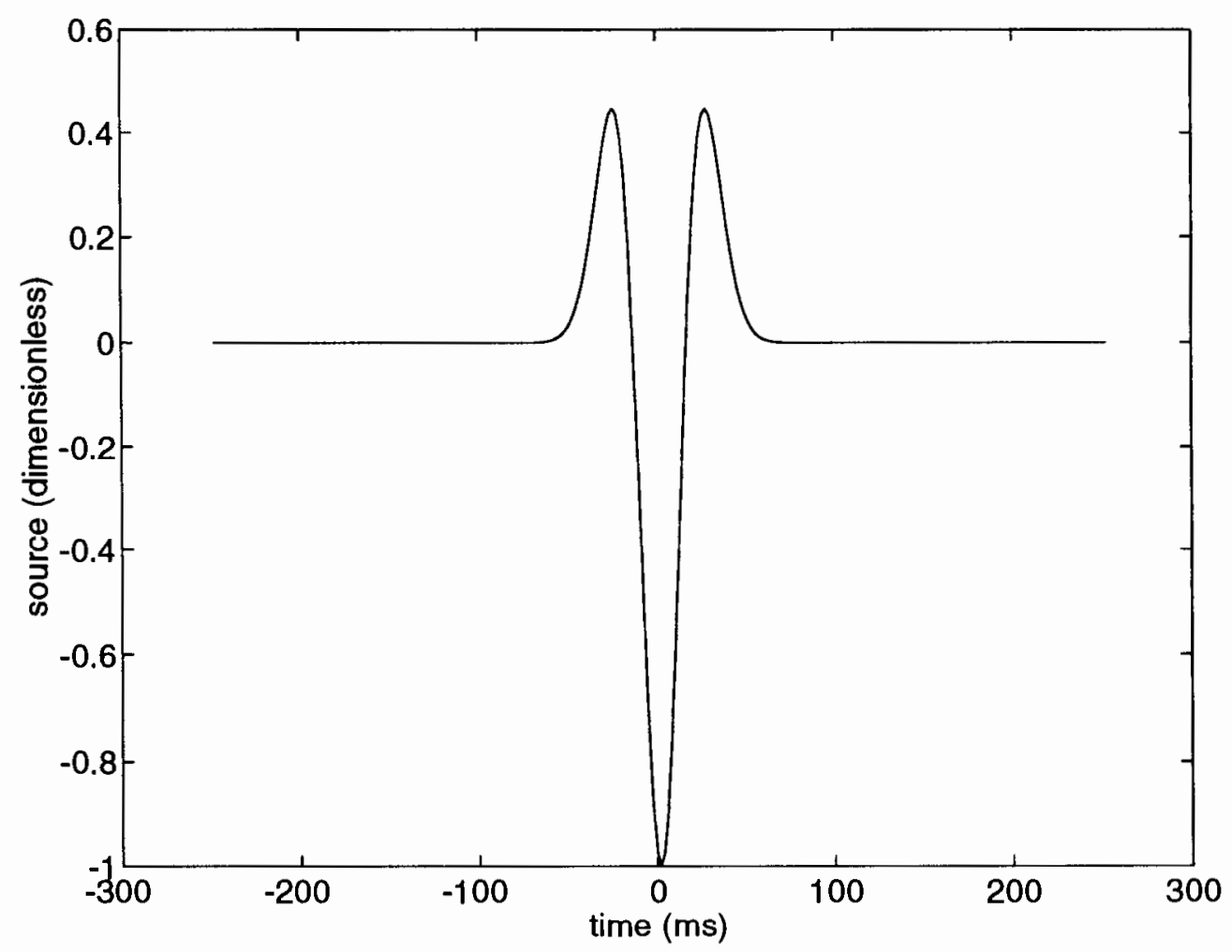

Figure 4.3: Initial guess for the source, an inverted $15 \mathrm{~Hz}$ Ricker wavelet centered at $0 \mathrm{~ms}$. 


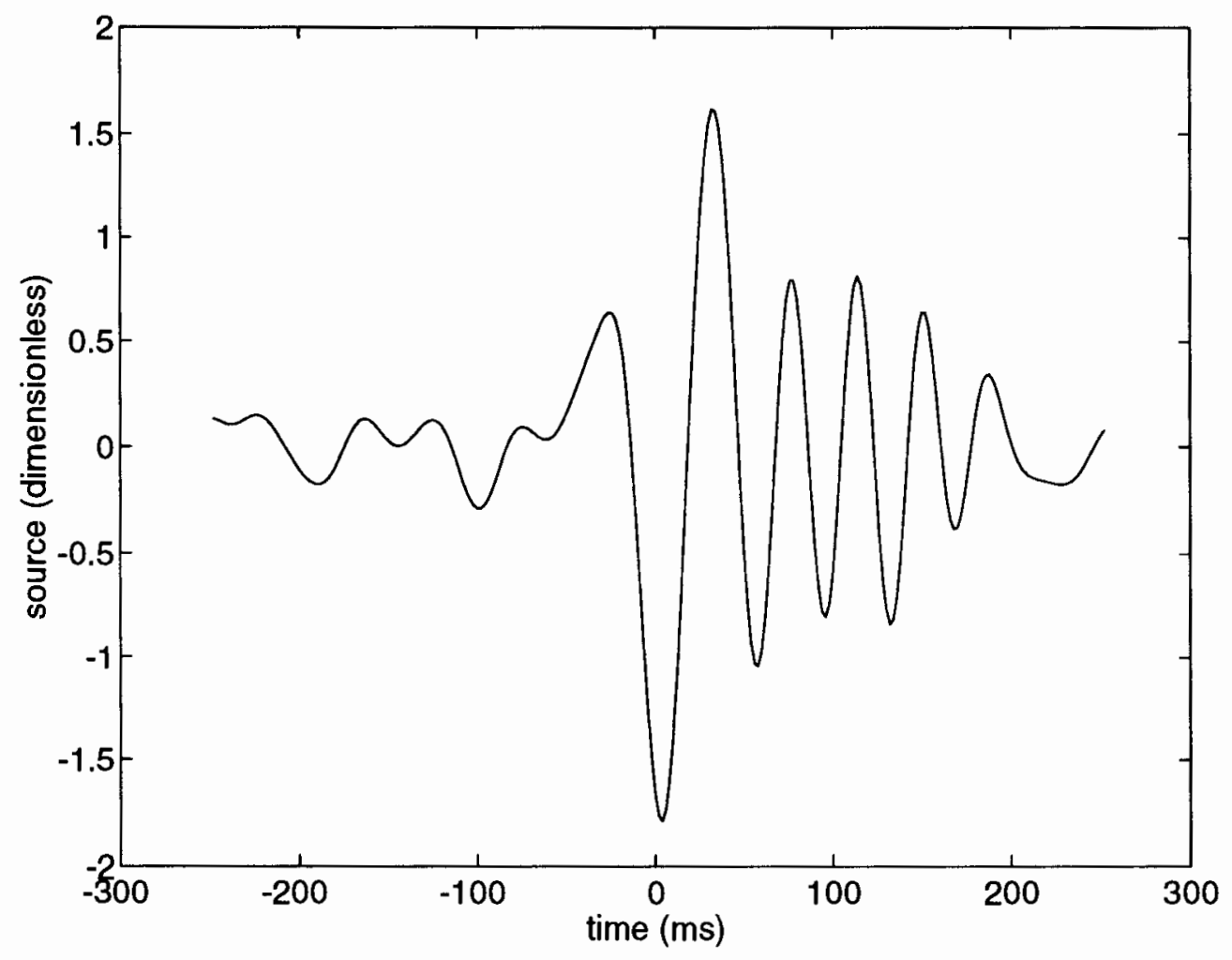

Figure 4.4: Source inversion result from the third round of inversion jobs. Already energy has begun to move towards positive time, the location of the true source. 


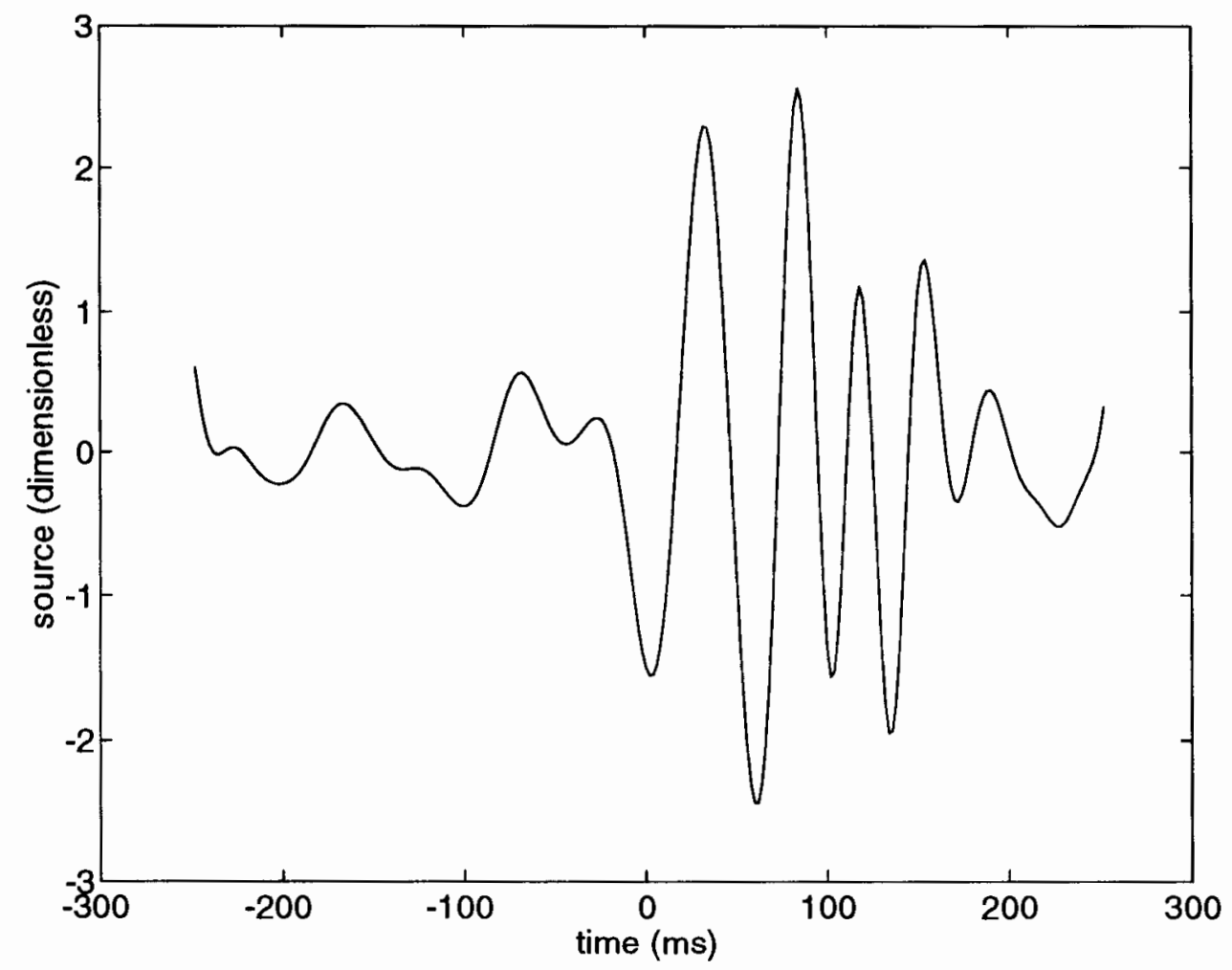

Figure 4.5: Source inversion result from the thirteenth round of inversion jobs. 


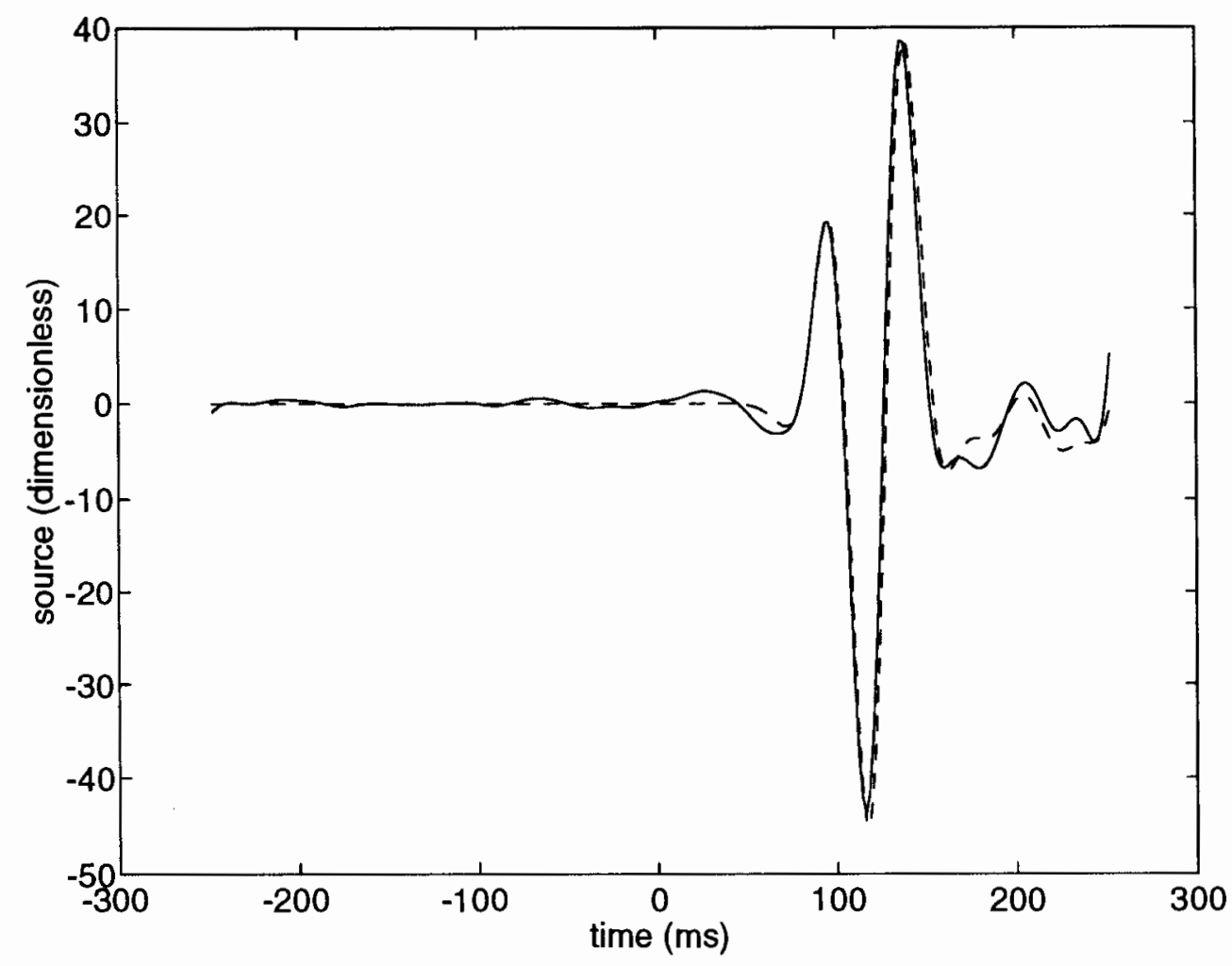

Figure 4.6: Solid line: Final inversion result for the source (iteration 60). Dashed line: Target source. Again, the final source estimate was scaled so the peaks for the two graphs are the same. 